\title{
Radioactivity: Old and New*
}

\section{By the Right Hon. LoRd Rutherford, o.M., F.r.s.}

$I^{n}$ WAS much honoured by the invitation to give the first course of the Joly Memorial Lectures. As is well known, Joly was for many years not only intensely interested in the problems of radioactivity, but also made numerous original and important contributions to our knowledge of this subject. When the large and continuous emission of heat from the radioactive bodies was made clear in 1903, Joly's alert and original mind was at once attracted to the problem of the effect of this steady generation of heat by the radioactive bodies present in the earth's crust on the geological history of our planet. To obtain reliable data, he devised simple but ingenious methods for measuring the amount of the primary radioactive bodies, uranium and thorium, in typical rocks constituting the earth's crust. He was the first to point out the far-reaching significance of this small but steady supply of heat on the internal temperature gradient of the earth, resulting in violent movements in the earth's crust. Indeed, he was of opinion that the rise and fall of continents and the elevation of mountain chains were intimately connected with the heating effect of radioactive bodies over long intervals of time. A fascinating account of these bold and original ideas has been given by Joly in his books and papers.

Of all the contributions made by Joly in the field of radioactivity, the discovery of the origin of the pleochroic haloes, observed in certain kinds of mica, has left the strongest impression on my mind. It required not only an acute and original mind but also a touch of real genius to connect these minute baloes, the origin of which had long been a mystery, with the effects of radioactive transformations. Joly, as the result of an intensive investigation, was able to prove that the darkening of the mica halo was due to the $\alpha$-particles liberated over ages from a minute inclusion containing either uranium or thorium. In a well-developed halo, a series of rings was observed, each of which corresponded in radius to the range of one of the groups of $\alpha$-particles liberated from the radioactive material. In this way, he was able to show that the processes of radioactive transformation were the same hundreds of millions of years ago as to-day. In some very old micas, Joly observed an inner ring corresponding to $\alpha$-particles of much shorter range than any observed from the known radioactive bodies. To account for it he postulated the earlier existence of an unknown radioactive element which he provisionally named 'hibernium'. The

\footnotetext{
* From the first Joly Memorial Lecture delivered at Trinity College, Dublin, on January 23, 1935.
}

researches of Hevesy in the last few years indicate that this ring is probably due to a known element, samarium, which has recently been found to be weakly radioactive. These haloes are produced by an exceedingly minute quantity of uranium or thorium as in inclusion in the mica. Probably the emission on an average of one $\alpha$-particle every one hundred years continuing through geologic ages would give rise to a discernible halo.

I could give many more illustrations from other fields of inquiry which equally show that Joly possessed to a marked degree that rare quality of originality and vision characteristic of the greatest investigators of the past.

The property of spontaneous radioactivity is shown to the most marked extent by the heaviest elements, uranium and thorium, and the products which arise from their transformation. Only a few lighter elements show this property and those only to a feeble degree. We now know that the property of radioactivity is a sign of the instability of the atoms concerned. Take, for example, the best known radioactive element, radium. For some unknown reason, each second a minute fraction of the radium atom becomes unstable and breaks up with explosive violence, a fragment of the atom-an $\alpha$-particle-being hurled out with high speed. As a consequence of these atomic transformations, a new type of atom is formed-the radium emanation-which in turn is unstable, breaking up rapidly with the liberation of an $\alpha$-particle. The process of transformation once started proceeds through a number of successive stages, finally ending in the formation of a stable and non-radioactive isotope of lead. The successive transformations of uranium lead to the formation of twelve distinct types of radioactive element, and in all more than thirty of these radioactive elements are known. Each of these elements suffers transformation according to a definite law but at a different and characteristic rate. For example, radium is half transformed in 1600 years, the radium emanation in 3.82 days, and its succeeding product radium $\mathrm{A}$ in 3 minutes.

In most of the transformations, $\alpha$-particles are alone expelled, but in a few cases the transformation is accompanied by the expulsion of $\beta$-rays, which are swift electrons. The mass of the atom is not sensibly changed by the expulsion of such a light particle, but the properties of the resulting atom are entirely changed. There is one important feature which distinguishes $\beta$-ray from $\alpha$-ray transformations; all the $\alpha$-particles escape with 
identical speed and thus the transformation of each atom leads to the liberation of an equal quantity of energy. In the case of a $\beta$-ray transformation, one $\beta$-particle is expelled per atom, but the speed of the $\beta$-particle varies widely from atom to atom. If this be the case, one atom loses more energy than another, and we should consequently expect the energy content of the new atoms formed to differ. There is, however, no evidence that such differences of energy exist, and indeed when an $\alpha$-ray body is formed by a $\beta$-ray transformation, the $\alpha$-particles are again expelled with identical speeds. This difference between $\alpha$ - and $\beta$-transformations raises great difficulties and no satisfactory explanation is as yet forthcoming. It has been suggested either that the conservation of energy cannot be applied to such $\beta$-transformations, or that some of the energy from the atom is carried off in the form of an undetectable particle of very light mass called the 'neutrino'. We shall see that the same problem arises in the transformation of radioactive bodies which can be produced by artificial methods.

It is desirable at this stage to say a word about the nuclear structure of atoms. At the centre of each atom is a minute but massive nucleus which carries a resultant positive charge. This charge controls the number and arrangement of the electrons which surround the nucleus. The properties of an atom are thus defined by a whole number which represents the number of units of charge carried by the nucleus. This fixes the number and order of the elements, but we now know that there are many species of the same element, defined by its nuclear charge, which have different masses. The number of these isotopes of an element varies; some elements like aluminium are apparently simple, but others like tin have a dozen isotopes varying in mass over a wide range.

The appearance of radioactivity results from the spontaneous transformation of the minute nucleus of an atom. The expulsion of an $\alpha$-particle carrying two positive charges lowers the charge on the nucleus of the residual atom by two units, while the expulsion of a $\beta$-particle raises it by one.

If we are to effect the transformation of an element, we must in some way alter its mass or its charge. This can be done by adding or subtracting a particle, whether charged or uncharged, from the nucleus. This is easy in imagination but difficult in practice. The most effective way so far found is the bombardment method, involving the entry of a foreign particle into the structure of the nucleus and in some cases leading to the loss of a charged particle from the nucleus. We shall illustrate these different types of transformation by considering a few of the simpler typical cases.
The first proof of the artificial transformation of an atom was given in 1919, when it was found that the bombardment of the gas nitrogen by fast $\alpha$-particles gave rise to the liberation of a number of fast protons which could only come from a disintegration of the nitrogen nucleus. It is now clear that about one $\alpha$-particle in 100,000 comes close enough to a nitrogen nucleus to enter its structure. The $\alpha$-particle is captured and momentarily forms a new nucleus of charge 2 units higher and of mass 4 units greater. This newly formed nucleus is unstable and breaks up with explosive violence, expelling a proton in the process. The mechanism of the reaction is given below:

$$
{ }_{7} \mathrm{~N}^{14}+{ }_{2} \mathrm{He}^{4} \rightarrow{ }_{9} \mathrm{~F}^{18} \rightarrow{ }_{8} \mathrm{O}^{17}+{ }_{1} \mathrm{H}^{1} .
$$

The resulting stable nucleus is an isotope of oxygen of mass 17, which we now know to exist in small quantity in ordinary oxygen. About twelve light elements can be disintegrated by $\alpha$-particles, and in all cases protons of characteristic speeds are emitted; the general mechanism of the reaction is probably similar, leading in all cases to the emission of a proton and the formation of a new but stable nucleus of mass 3 units greater.

During the last few years, another important type of transformation has been brought to light in which a new and strange type of particle called the neutron is expelled. The neutron, as its name implies, carries no electric charge, but has a mass about equal to that of the proton. Since it has no charge, it does not ionise the gas in its path and consequently shows no track in an expansion chamber. At rare intervals in its passage through a gas, it hits the nucleus of an atom in its path and shoots it forward with high speed. This struck nucleus is charged and ionises the gas, so that its track through the gas is shown in a cloud chamber. While these 'knock-ons' are comparatively rare, they form a very convenient method of detecting the presence of the invisible neutron and forming an idea of its velocity. One of the best ways of producing a supply of neutrons is to bombard the element beryllium with fast $\alpha$-particles. The $\alpha$-particle is captured and the resulting nucleus of carbon 13 breaks up into a carbon nucleus of mass 12 , while a fast neutron is expelled. The mode of the reaction is shown below :

$$
{ }_{4} \mathrm{Be}^{9}+{ }_{2} \mathrm{He}^{4} \rightarrow{ }_{6} \mathrm{C}^{13} \rightarrow{ }_{6} \mathrm{C}^{12}+\text { neutron }{ }^{1} .
$$

We are now able to produce neutrons in a variety of ways, not only by the action of $\alpha$-particles, but also by bombarding different elements by protons and deuterons.

Still another striking type of transformation can be produced by $\alpha$-particles, resulting in the artificial production of new radioactive bodies. In the transformations previously considered, the 
residual nucleus is a known type of stable nucleus, and is non-radioactive. A radioactive nucleus is an unstable type of nucleus which breaks up with the emission of fast particles, ultimately forming another stable nucleus. The production of an artificial radioactive body was first noted by M. and Mme. Curie-Joliot by bombarding boron with fast $\alpha$-particles. After bombardment for a few minutes, the boron continued to show a marked activity for an hour or so after it was removed from the bombarding source. This activity decayed in exactly the same way and according to the same laws as the ordinary well-known radioactive bodies, but the type of particle emitted was quite different. Strange to say, it was found that high-speed positive electrons, or positrons, were emitted, and not $\alpha$-particles or negative electrons such as appear in the spontaneous transformations of the radioactive bodies uranium and thorium. In order to account for the production of a radioactive element by bombarding boron with fast $\alpha$-particles, it seems clear that the transformation occurs in two stages according to the scheme below :

$$
\begin{aligned}
{ }_{5} \mathrm{~B}^{10}+{ }_{2} \mathrm{He}^{4} & \rightarrow{ }_{7} \mathrm{~N}^{13}+\text { neutron }{ }^{1} \\
{ }_{7} \mathrm{~N}^{13} & \rightarrow{ }_{6} \mathrm{C}^{13}+{ }_{1} \text { positron. }
\end{aligned}
$$

In the first stage, the $\alpha$-particle is captured by the boron nucleus forming an unstable isotope of nitrogen, $\mathrm{N}^{13}$, together with the liberation of a fast neutron. This isotope of nitrogen does not exist in Nature and is transformed slowly into the stable isotope of $\mathrm{C}^{13}$ by the expulsion of a positive electron. The activity of the radioactive body $\mathrm{N}^{13}$ decays to half value in about 14 minutes. By using the activity of this body as an indicator of its presence, it was shown by chemical methods that the radioactive body was an isotope of nitrogen. In a similar way, by bombarding other elements, radioactive isotopes of phosphorus and silicon could be formed, each with a characteristic period of decay.

This new discovery was rapidly followed up in a number of directions. Prof. E. Fermi and his collaborators in Rome found that a large number of these radioactive bodies could be formed when the elements were bombarded by neutrons derived from the action of $\alpha$-rays on beryllium. On account of the absence of charge, the neutron is able to enter the structure of even a heavy element, where the $\alpha$-particle on account of its charge is ineffective. Even the heaviest element, uranium, when bombarded by neutrons, gives rise to at least four new distinctive types of radioactive bodies, with half periods of 15 seconds, 40 seconds, 13 minutes and 100 minutes. The actual nature of the transformations involved has only been studied in a few cases. In all known cases, the neutron is cap- tured; sometimes a heavy particle, an $\alpha$-particle or proton, is emitted, but generally a higher isotope of the element is formed which may be unstable and emit negative electrons. It is worthy of note that all the radioactive bodies produced in this way break up with the liberation of fast negative electrons and not positive electrons, as in the original cases found by the Joliots. Cockeroft, Lawrence and others have found that bombardment of certain elements by fast hydrogen ions also gives rise in some cases to the production of new radioactive bodies.

It seems clear from these results that we are able greatly to increase our knowledge of the isotopes of the elements. None of these radioactive bodies are found in Nature, but represent unstable types of isotopes with a very limited life. During the last few months, it has been found that the efficiency of the process can be increased in some cases about a hundred times or more by slowing down the neutrons. This can be done by passing them through hydrogen or a solid material like water or paraffin which contains hydrogen.

We have so far spoken of the transformations brought about by the swift $\alpha$-particles spontaneously expelled from radioactive substances, and the neutrons which arise from the bombardment of certain elements by $\alpha$-particles. Another notable advance has been made by the use of bombarding particles produced artificially in the electric discharge and speeded up by appropriate methods. This involves complicated apparatus and in general the use of high potentials of the order of one million volts. In this way, copious streams of fast particles can readily be produced, corresponding in number to the $\alpha$-particles expelled from thousands of grams of radium. Cockeroft and Walton were the first to show that transformation effects on a comparatively large scale could be produced by bombarding light elements like lithium and boron by fast protons. Time does not allow me to consider the mechanism of these transformations, which are different for each isotope of the same element. Transformation only appears to occur when the proton is captured by the nucleus, which then breaks up with explosive violence. Similar but different effects are observed when the ions of heavy hydrogen of mass 2 are used as bombarding particles. I would like, how. ever, to refer to recent experiments in which it has been shown that artificial radioactive elements can also be produced by these new methods. Cockeroft and Walton and others have shown that ordinary carbon, whether bombarded by $\mathrm{H}^{1}$ or $\mathrm{H}^{2}$ particles, gives rise to the production of a new radioactive substance which decays to half value in about 11 minutes, emitting positive electrons in the process. It is believed that the 
radioactive body formed in both cases is $\mathrm{N}^{13}$, which is transformed into the isotope of carbon $\mathrm{C}^{13}$ by the emission of a positron. It is not yet settled whether this radio-nitrogen differs from that formed by $\alpha$-rays, where the half period seems to be longer, namely, 14 minutes.

It seems likely that when very intense streams of still swifter particles are available, radioactive bodies of strong intensity may be produced by these artificial methods. It may be also that some of the bodies produced in these ways may give rise to a succession of changes such as is characteristic of the heavier elements uranium and thorium.

Sufficient, I think, has been said to illustrate the extraordinarily interesting results obtained in this fascinating field of inquiry. We can build heavier elements from lighter, and break up other atoms into fragments, and produce novel radioactive elements by the score. This new field of what may be called nuclear chemistry is opening up with great rapidity. Much work, often of a difficult technical character, will be required to prove the exact nature of any of the transformations which have been observed, but a very promising beginning has been made. Future work may disclose many surprises, for new and unsuspected particles may come to light. In any event, we are entering a no man's land with the ultimate hope of throwing light on the way atoms are built up from simpler particles.

\section{A New "Nomenclator Zoologicus"}

$\mathrm{F}^{\mathrm{n}}$ ROM the time that the tenth edition (1758) of the "Systema Naturæ" of Linnæus, which established the binomial system, was recognised as the basis of the nomenclature of animals, systematists have always had to face the difficulty of ascertaining what names have been used for genera. Since the same name cannot be validly used more than once in the whole of zoology, it is obvious that unless this information is reasonably accessible, many homonyms are inevitably created and themselves add to the confusion. Moreover, even when the existence of a name is known, it is often by no means an easy matter to find the original reference to it or to ascertain its position in the animal kingdom. Before describing the situation at the present day, it may be as well briefly to review the attempts that have been made to solve these problems.

The first Nomenclator to be published was that of Agassiz, which appeared between 1842 and 1846 ; it was followed by that of Marschall, which covered the period 1846-68 and was published in 1873. In 1864 there appeared the first volume of the "Zoological Record" which, however, in its earlier years, did not always include an annual list of new genera. The names in all these, with many others, were collected together by Scudder, who attempted a list of all published genera from Linnæus to 1879 , and his work, which was published in 1882-84 as Bulletin No. 19 of the U.S. National Museum, is the only list of its kind that has yet been completed. It contains about 80,000 names. Supplements to Scudder's work are represented by the two volumes, edited by Waterhouse and published by the Zoological Society of London, of the new genera contained in the "Zoological Record" with some additions. These volumes together contain some 62,000 names, and cover the periods 1880-1900 and 1901-10 respectively.
The new generic names in the "Zoological Record", which average about 2,000 per annum, have not been collected together since that date.

Sherborn's famous "Index Animalium", which was published between 1902 and 1933 and deals with both generic and specific names, though marvellously complete, only goes up to the year 1850. There also began to be published in 1926, under the auspices of the Prussian Academy of Science, an ambitious work entitled "Nomenclator animalium generum et subgenerum". If circumstances had permitted this to be carried through as originally planned, it would have been a very important contribution to the problem for the period that it dealt with. Though it appears to cover the ground with relatively few omissions up to about 1909 and it purports to include all names up to 1922 , those dealing with the later years are taken almost entirely from the annual indexes to the "Zoological Record". The choice of the date 1922 was peculiarly unfortunate, because the "Zoological Record" was necessarily far from complete in the War years and those immediately following, with the result that it omits the very numerous names that were actually published during 1914-22 but were only found and recorded afterwards. Furthermore, only about three quarters of this German work have yet appeared, although it has been nine years in course of publication. Its use is therefore very limited, and it has the added disadvantage of being extremely expensive.

The present position is, therefore, that a system. atist who wishes to erect a new generic name finds it almost impossible, even at the expense of much labour, to satisfy himself that any given one has not been used already. Not only are his sources of information very scattered and far from complete, but also many of them are rare and expensive. 